\title{
Die Versprechen der Demokratie und die Grenzen der Deliberation
}

\begin{abstract}
Kurzfassung
Der Artikel wendet sich gegen eine deliberative Neubeschreibung der Demokratie, wie sie gegenwärtig insbesondere im Kontext der Diskussion über Demokratie jenseits des Nationalstaates zu beobachten ist. Ausgangspunkt der Kritik ist die Überlegung, dass sich zwar kein Kern der Demokratie bestimmen, die Wirkmächtigkeit der demokratischen Idee sich aber anhand von zwei Versprechen festmachen lässt. Dabei handelt es sich um das prozedurale Versprechen der gleichen Teilhabe am politischen Prozess und das substantielle Versprechen einer Angleichung der sozialen Lebensverhältnisse. Es wird argumentiert, dass ein deliberatives Verständnis von Demokratie diese beiden Versprechen zu verletzen droht. Dies tritt besonders bei einer deliberativen Deutung der europäischen Union hervor. Im letzten Teil wird demgegenüber der Vorschlag formuliert, nicht länger politische Systeme sondern jene Handlungsweisen als demokratisch zu bezeichnen, die sich gegen Verletzungen der demokratischen Versprechen richten.
\end{abstract}




\section{Inhalt}

1. Eine demokratietheoretische Sackgasse 270

2. Die Versprechen der Demokratie 272

3. Die demokratischen Grenzen der Deliberation 274

4. Konzeptionelle Alternativen 278

a) Renationalisierung der Politik 279

b) Postdemokratie 281

c) Demokratie als Widerspruch 283

\section{Eine demokratietheoretische Sackgasse}

Betrachtet man den demokratietheoretischen Diskurs der Gegenwart, so springt die Dominanz deliberativer Ansätze ins Auge. Zwar existiert weiterhin eine Vielzahl konkurrierender Paradigmen, doch der Mainstream der Demokratietheoretiker schwimmt im deliberativen Wasser. Bezeichnend hierfür ist etwa folgende Aussage von Philip Pettit: „It is now widely accepted as an ideal that democracy should be seen as deliberative as possible“ (2004: 52). Auch wenn in diesen Worten gewiss eine theoriepolitische Übertreibung liegen mag, so geben sie nichtsdestotrotz ein Bild von der derzeitigen Hegemonie deliberativer Ansätze.

Dem stehen Autoren wie Michael Walzer (1999), Alexander Somek (2009) oder auch Marcus Höreth (2009) gegenüber, die sich gegen eine deliberative Neubeschreibung des Demokratiekonzeptes wenden. Eine politische Willensbildung von unten nach oben - als normativer Kern der Demokratie - sei nur im Rahmen von Verfahren möglich, die die Wahl und eben auch die Abwahl der politischen Entscheidungseliten gewährleisten. Von diesem Input-orientierten Verständnis hätten sich aber - so Höreth in erfrischender Zuspitzung die Vertreter deliberativer Demokratietheorien insbesondere im Kontext der Diskussion zur europäischen Governance größtenteils verabschiedet. Deliberative Arenen wie die der europäischen Komitologie oder der europäischen Gerichtsbarkeit würden zu einem Demokratiesurrogat erhoben, die „fast schon einen Legitimationsüberschuss genießen“ (Höreth 2009: 311), gerade weil sie auf eine Partizipation der Bürgerinnen und Bürger verzichten würden. Insgesamt kommt Höreth zu dem Urteil, dass die deliberative Demokratietheorie sowohl „überangepasst“ als auch „realitätsentrückt" sei. Sie sei überangepasst, insofern sie demokratische Defizite zu einem ,normativen Mehrwert“ umdeu- 
tet, und realitätsentrückt, insofern sie Entscheidungsprozesse im europäischen Mehrebenensystem als deliberativ adelt, die lediglich „in Ausnahmefällen“ (Höreth 2009: 307) den Idealen der deliberativen Demokratietheorie entsprechen würden.

In einer Replik auf Höreth hebt Jürgen Neyer demgegenüber die Stärken der deliberativen Theorie hervor und spricht von einem „Elend der orthodoxen Demokratietheorie" (2009: 336). Dieses bestünde vornehmlich darin, dass sie sich der Aufgabe, ,den Begriff der Demokratie auf die empirischen Bedingungen des 21. Jahrhunderts einzustellen“ (Neyer 2009: 336), verweigern würde. Der „orthodoxen Demokratietheorie“ läge ein ,normativer Nationalismus" zugrunde, der nicht nur ,historisch überholt" sei, sondern auch jegliche „intellektuelle Kreativität" vermissen lasse (2009: 336). Demgegenüber weise die deliberative Theorie mit ihrer Metanorm des „Rechts auf Rechtfertigung“ (Forst 2007) einen produktiven und empirisch durchaus gesättigten Ausweg aus dem „nationalstaatlichen Container“ (Neyer 2009: 331). Neyer verweist insbesondere auf Forschungen zum zunehmend argumentativen Charakter politischer Prozesse im Rahmen der Europäischen Union, aber auch im Kontext der internationalen Politik.

Im Folgenden möchte ich nicht der empirischen Frage nach dem tatsächlichen oder auch nur vermeintlichen deliberativen Gehalt europäischen oder internationalen Regierens nachgehen. Im Zentrum steht vielmehr die Frage nach den demokratietheoretischen Grenzen der von Neyer eingeforderten ,intellektuellen Kreativität". Lässt sich der Demokratiebegriff von seinem Entstehungskontext lösen und auf neue Räume, aber auch neue Verfahren der Entscheidungsfindung übertragen? Oder setzt die Idee und die Praxis der Demokratie, wie Höreth behauptet, eine politische Willensbildung von unten nach oben oder zumindest Verfahren der effektiven Kontrolle der politischen Entscheidungsträger voraus? Und wenn das der Fall sein sollte, wie könnte eine nicht rückwärtsgewandte Antwort auf die postnationale Konstellation aussehen?

Diesen Fragen soll in drei Schritten nachgegangen werden. Zunächst geht es darum, an zwei zentrale Versprechen der Demokratie zu erinnern, nämlich das Versprechen der möglichst breiten Beteiligung des Demos einerseits und das Versprechen, dass demokratische Beteiligung zu einer sozialen Inklusion führt, andererseits (2). Daran anschließend zeige ich, dass die gegenwärtige Praxis der Deliberation, gerade im Kontext der Europäischen Union, diese beiden Versprechen unterwandert (3). In einem letzten Schritt werde ich drei Auswege aus der gegenwärtigen demokratietheoretischen Sackgasse, die in der 
Kontroverse zwischen Höreth und Neyer hervortritt, diskutieren: die Idee einer Renationalisierung politischer Entscheidungsprozesse, der Verzicht auf zu hohe normative Erwartungen im Anschluss an Überlegungen zur „Postdemokratie" von Colin Crouch sowie der Vorschlag, als demokratisch den Widerspruch gegenüber jenen politischen Entscheidungen und Strukturen auszuzeichnen, die den Versprechen der Demokratie entgegenstehen (4).

\section{Die Versprechen der Demokratie}

In seinem jüngsten Buch zur Demokratischen Legitimität beklagt Pierre Rosanvallon die "missbräuchlichen Verwendungen und Verwechslungen“ des Demokratiebegriffs, die dessen Vagheit geschuldet seien. Auch der Verweis auf Formeln wie „Herrschaft des Volkes“ würde nicht weiterhelfen und es gäbe „kaum ein Wort der politischen Sprache, dessen praktische Definition so vielen Variationen ausgesetzt ist". In der Konsequenz erscheint der Demokratiebegriff „nicht mehr als eine Lösung, sondern als ein Problem. Das Gute und Vage haben in ihm stets koexistiert" (2010: 279).

Die von Rosanvallon beklagte Kombination aus Vagheit und einem überwiegend affirmativen Begriffsgebrauch zeigt, dass ,Demokratie ‘ zu jenen politischen Wörtern gehört, die ihrem Wesen nach umstritten sind. Als „essentially contested concepts" können im Anschluss an Gallie (1956) jene Begriffe verstanden werden, die folgende Merkmale aufweisen: Sie sind in einem erheblichen Umfang normativ aufgeladen, es gibt verschiedene, sich historisch wandelnde Vorstellungen darüber, worin deren normativer Kern besteht, und es existiert ein unterschiedlicher Gebrauch dieser Konzepte. So ist es wenig verwunderlich, dass die Verwendungsweisen und Definitionen von ,Demokratie' selbst für Experten der Demokratietheorie nicht mehr zu überblicken sind. Und offenbar muss mit dem Eingeständnis der grundlegenden semantischen Umkämpftheit auch jedes Unterfangen scheitern, dem Begriff, Demokratie' einen dauerhaften Bedeutungskern einzuschreiben. Das ändert jedoch wenig an seiner Attraktivität. Im Gegenteil, gerade seine Vagheit macht ihn so vielseitig einsetzbar. Durch den Gebrauch des Demokratiebegriffes - wie auch der Begriffe der Freiheit und der Gerechtigkeit - wird ein politischer Mehrwert erzeugt (Connolly 1993: 29 ff.). Wenn ein Regime oder eine Praktik als ,demokratisch' oder , undemokratisch' bezeichnet wird, so ist damit immer auch eine begriffspolitische Dimension verbunden, die die entsprechenden Institutionen und Praktiken auszuzeichnen oder zu diskreditieren trachtet. Daher lässt sich eine ständige theoriepolitische Auseinandersetzung mit der Demokratie beo- 
bachten, die deren normativen Gehalt wie auch ihre zentralen Praktiken immer wieder aufs Neue festzulegen versucht.

Doch was macht den Begriff der Demokratie so attraktiv? Was ist das ,Gute', das durch die Verwendung von ,Demokratie' hervorgerufen wird? Meine These ist, dass mit dem modernen Begriff der Demokratie, wie er sich seit dem 19. Jahrhundert etabliert hat, zwei zentrale Versprechen verbunden sind. ${ }^{1}$ Es handelt sich um Versprechen der Demokratie, die zwar miteinander verschränkt sind, sich aber analytisch unterscheiden lassen, nämlich das prozedurale Versprechen der gleichen Teilhabe am politischen Prozess und das substantielle Versprechen einer Angleichung der sozialen Lebensverhältnisse. Wohl kein politischer Denker hat diesen Zusammenhang so sehr ins Zentrum der Analyse der modernen Demokratie gerückt wie Alexis de Tocqueville in seinem Buch Über die Demokratie in Amerika (1835/40).

Für Tocqueville ist die Demokratie in den Vereinigten Staaten in erster Linie eine Gesellschaftsform, die durch ein hohes Maß von sozialer Gleichheit bestimmt ist. Diese zeigt sich in dem Fehlen von Standesunterschieden, aber auch in einer Angleichung der gesellschaftlichen Lebensumstände, welche Tocqueville auf den natürlichen Reichtum Nordamerikas und ein Erbrecht zurückführt, welches eine gleichmäßige Güterverteilung erzwingt. Doch ist die soziale Gleichheit ohne die gleichzeitige Verwirklichung einer politischen Gleichheit für ihn nicht vorstellbar. ,Selbstverständlich durchdringt die Gleichheit schließlich das politische wie das übrige Leben. Die Menschen können unmöglich in einer einzigen Hinsicht immer ungleich, in allen anderen gleich sein" (1835/40: 61). Es ist hier nicht der Ort, um auf Tocquevilles detaillierte Analysen des Bedingungsverhältnisses von sozialer und politischer Gleichheit einzugehen. Entscheidend ist aber der konstitutive Zusammenhang von beiden Dimensionen der Demokratie, auf den er verweist.

Freilich hatte Tocqueville immer auch seine französische Heimat im Sinn, wenn er gleich zu Anfang seines Buches, dessen erster Band 1835 erschienen ist, feststellt, dass der Demokratie die Zukunft gehöre, die Geschicke der Menschen ohne sie nicht mehr denkbar seien. Im weiteren Verlauf der europäischen Geschichte wurde die Etablierung der politischen Gleichheit im Sinne der Volkssouveränität je nach Perspektive als die Gefahr oder als die Chance der Angleichung der sozialen Lebensverhältnisse gesehen. Davon zeugen auf der einen Seite die konservativen Kritiker einer Ausdehnung des Wahlrechts und

1 Zur Geschichte des modernen Demokratiebegriffs vgl. Dunn (2005) und Rosanvallon (2009). 
auf der anderen Seite sozialistische und später sozialdemokratische Programmatiken, die sich von der Ausweitung des Wahlrechts eine Aufhebung der Klassengesellschaft versprochen haben. Mit der langsamen Durchsetzung des allgemeinen Wahlrechts sind im 19. und frühen 20. Jahrhundert schließlich jene Hoffnungen geweckt worden, die in der politischen Praxis durch die periodische Wahl der Repräsentanten und ergänzender Verfahren der Partizipation zu erfüllen sind.

Normativ betrachtet, kommt in den beiden Versprechen der Demokratie, also das prozedurale Versprechen der gleichen Teilhabe am politischen Prozess und das substantielle Versprechen, durch demokratische Verfahren die Geschicke der Gesellschaft in Richtung auf mehr soziale Egalität bestimmen zu können, das moderne Ideal der Autonomie zum Ausdruck: die Idee einer positiven Freiheit der Selbstgesetzgebung und die Idee einer Person, die sich in ihren Lebensbezügen gerade nicht in Abhängigkeit von anderen Personen oder anonymen Mächten - etwa den Schwankungen des Arbeitsmarktes - erfährt (Christiano 1996).

Die Rede von den zwei zentralen Versprechen beansprucht nicht, einen semantischen Kern des Demokratiebegriffs festzulegen. Ein solches Vorgehen würde dessen essentiell umstrittenen Charakter widersprechen. Der Anspruch ist insofern bescheidener, als lediglich die Attraktivität des Wortes ,Demokratie', die sich seit der Mitte des 19. Jahrhunderts nachweisen lässt, erklärt werden soll. Die hier vorgeschlagene These lautet, dass die Attraktivität aus den durch seinen Gebrauch evozierten beiden Versprechen resultiert. Wenn nun im folgenden Abschnitt argumentiert wird, dass deliberative Theorien sich von diesen beiden Versprechen der modernen Demokratie entfernen, dann soll damit nicht behauptet werden, dass damit undemokratische Intentionen verbunden seien. Behauptet wird aber, dass deliberative Theorien in ihrer Übertragung auf die Analyse und Interpretation realer politischer Prozesse den Versprechen nicht gerecht werden und mithin Gefahr laufen, gerade jene Prozesse, die die prozedurale und die soziale Gleichheit unterlaufen, als ,demokratisch`zu verklären.

\section{Die demokratischen Grenzen der Deliberation}

Deliberative Ansätze befinden sich nicht nur im Zentrum der gegenwärtigen Diskussion, sie beanspruchen darüber hinaus auch in besonderer Weise zu einer kreativen Weiterentwicklung der Demokratie beizutragen. Deren akademische Attraktivität besteht insbesondere in dem Versprechen, Demokratie und 
Rationalität zu versöhnen, und zwar durchaus im Sinne der Gemeinwohlorientierung. In einem öffentlichen und inklusiven Diskurs sollen all jene Gründe aussortiert werden, die nicht verallgemeinerungsfähig sind. Die entsprechenden Prozeduren sollen nicht allein zur Aufklärung über die jeweiligen Präferenzen beitragen, sondern als eine Art moralisches Reinigungsmittel (Goodin 1986) dienen. Die deliberative Demokratie wird von ihren Verfechtern zudem als eine Art Allheilmittel gegen die gegenwärtige Malaise demokratischer Praxis, die sich in zunehmender politischer Apathie und populistischen Tendenzen zeigt, angepriesen. Ihr Siegeszug resultiert aber vor allem aus einem neuen Fokus der demokratietheoretischen Debatte: der Frage nach den Möglichkeiten einer Demokratisierung supra-, trans- und internationalen Regierens. Argumentiert wird, dass ein deliberatives Verständnis die Perspektive eröffnet, Demokratie aus einem nationalstaatlichen Korsett zu befreien und dergestalt zu konzeptionalisieren, dass Formen postnationaler Legitimitätserzeugung in der komplexen Welt der globalen Governance ausgemacht werden können. Der hierfür entscheidende begriffsstrategische Schritt besteht - in einer viel zitierten Formulierung von Jürgen Habermas - darin, dass das demokratische Verfahren „seine legitimierende Kraft nicht mehr nur, und nicht einmal in erster Linie, aus Partizipation und Willensäußerung, sondern aus der allgemeinen Zugänglichkeit eines deliberativen Prozesses [bezieht], dessen Beschaffenheit die Erwartung auf rationale Ergebnisse begünstigt" (1998: 166). Ganz in diesem Sinne macht etwa James Bohman (2007) ,multiple demoi' aus, die in deliberativen Arenen jene verallgemeinerungsfähigen Gründe erzeugen, die eine demokratische Legitimität globalen Regierens garantieren sollen. ${ }^{2}$

Inzwischen mehren sich jene Stimmen, die zu Recht eine epistemische sowie eine elitäre Schlagseite der deliberativen Demokratietheorie hervorheben. ${ }^{3}$ Der „eigentümlich zwanglose Zwang des besseren Arguments“ (Habermas 1971: 137) legt einen wissenschaftlichen oder zumindest wissenschaftsanalogen Modus der Bearbeitung und Entscheidung politischer Fragen nahe. In der Konsequenz wird der Trend zur Verwissenschaftlichung der Politik legitimiert, gerade in hochkomplexen Politikfeldern wie denen der europäischen Finanzoder Verbraucherschutzpolitik. Insofern ist es nur ein kleiner Schritt von der Aufwertung einer epistemisch verstandenen Deliberation zur Treuhänderschaft wissenschaftlicher Experten im Rahmen der europäischen Komitologie auf

2 Zur Idee eines ,deliberativen Supranationalismus“ vgl. auch Schmalz-Bruns (1999).

3 Vgl. etwa Buchstein/Jörke (2003), Niesen (2008), Tucker (2008), Somek (2009) und Urbinati (2010). 
Kosten demokratisch legitimierter Akteure wie Parteien und Parlamente (Münch 2009: 599). Mit einem epistemischen Politikverständnis wird insbesondere die Rolle von Macht und Interessen verdeckt, denen sich auch Experten nicht entziehen können.

Die Praxis der Deliberation ist zudem sehr voraussetzungsvoll. Nicht alle Bürgerinnen und Bürger verfügen aber über jene Ressourcen, derer es für die erfolgreiche Partizipation an diskursiven Verfahren bedarf. Hierzu zählen neben Zeit und einer zumindest rudimentären Sachkenntnis auch rhetorische Fähigkeiten und ein selbstbewusstes Auftreten. Doch all diese Eigenschaften sind in spätmodernen Gesellschaften ungleich verteilt. Deliberative Arenen wie Bürgerversammlungen und Mediationsverfahren, aber auch die verschiedenen Organisationen der nationalen und internationalen Zivilgesellschaft werden vornehmlich von den gut ausgebildeten Mittelschichten dominiert. Damit wird aber lediglich ein Trend forciert, der sich bereits bei Wahlen beobachten lässt. Am politischen Prozess partizipieren Ober- und Mittelschichtangehörige zu einem signifikant höheren Anteil als die Angehörigen der Unterschicht (Walter 2009; Schäfer 2010). Insofern in deliberativen Verfahren besonders akademische Kompetenzen honoriert werden, wohnt dem deliberativen Demokratieverständnis, wenn schon nicht in der Theorie, dann aber in ihrer praktischen Umsetzung eine elitäre Tendenz inne.

Sowohl die epistemische als auch die elitäre Schlagseite können aus demokratietheoretischer Sicht nur so lange als unproblematisch angesehen werden, wie deliberative Verfahren konventionelle Beteiligungsformen lediglich ergänzen, nicht aber ersetzen sollen. Im nationalstaatlichen oder kommunalen Kontext können Ergebnisse der Deliberation durchaus in den politischen Prozess eingespeist werden, sie sollten jedoch nicht an den Parlamenten vorbei eins zu eins umgesetzt werden. Im Kontext der Diskussion um eine Demokratisierung supranationalen oder globalen Regierens ist die Rückbindung an eindeutige Legitimationsketten aber nur unzureichend gegeben. Im Gegenteil, viele Befürworter sehen deliberative Prozesse als eine Alternative zu Wahlen und Abstimmungen. Die Alternative wird darin gesehen, dass politische Eliten ihre Entscheidungen vor einer supranationalen Öffentlichkeit rechtfertigen müssen und dadurch zu einer gerechteren, weniger interessenbasierten Politik geradezu genötigt würden (Dryzek 2006; Bohman 2007). Dieser Deutung schließt sich auch Jürgen Neyer an. Zwar möchte er ,die Idee supranationaler Rechtfertigung“ (2009: 349) als einen „Idealtyp“ verstanden wissen, doch er sieht genügend entgegenkommende Tendenzen, die eine neue Form der Legitimität greifbar werden lassen. So stellt Neyer mit Blick auf die europäische Politik fest, 
dass diese, ,zu einem erstaunlich hohen Ausmaß von argumentativen und rechtfertigungsbasierten Prozessen geprägt ist" (2009: 340).

$\mathrm{Zu}$ bezweifeln ist jedoch die genuin demokratische Qualität der entsprechenden Rechtfertigungsprozesse. Zwar mag man in den europäischen Politiknetzwerken und der Komitologie durchaus einen hohen Grad an Deliberativität feststellen, aber das bedeutet nicht, dass damit auch ein hoher Grad an demokratischer, und das heißt immer auch inklusiver wie egalitärer, Beteiligung einhergeht. Im Gegenteil, wie Peter Niesen zeigt, lässt sich geradezu ein Auseinandertreten von Deliberation und Demokratie beobachten. Dann stellt sich aber in der Tat die Frage, ,warum Wert darauf gelegt wird, sozusagen zeremoniell weiterhin von ,Demokratischen Regieren in der EU' (Neyer 2005) zu sprechen" (Niesen 2008: 249). Kurzum, Deliberation wird zum Demokratiesurrogat erkoren und verletzt das prozedurale Versprechen der egalitären Beteiligung an politischen Entscheidungen. Der Verweis auf die deliberative Berücksichtigung der Interessen der so genannten "stakeholder“ sowie die Einbeziehung zivilgesellschaftlicher Akteure vermag diese Lücke insofern nicht zu füllen, als ein solches Modell, wie Ingeborg Maus konstatiert, in einen vordemokratischen Paternalismus zurückfällt: „Die klassische Garantie einer uneingeschränkten Öffentlichkeit und die Anerkennung zivilgesellschaftlicher Aktivitäten werden nicht mehr als notwendige Komplementärfunktionen der Volkssouveränität, sondern als deren Ersatz angesehen. Die Ansprüche bürgerlicher Freiheit werden so auf das Ausmaß zurückgestuft, das einst Kant als Mindestforderung an den aufgeklärten Absolutismus adressiert hatte" (2007: 355, Hervorhebung im Original).

Auch das substantielle Versprechen der Demokratie, das einer größeren sozialen Egalität, wird in einer deliberativen Deutung der europäischen Governance unterlaufen. In seiner Verteidigung des Rechts auf Rechtfertigung beruft Neyer sich auf die „außerordentlich wichtige Funktion“ des europäischen Rechts. Denn dieses garantiere, dass politische Präferenzen ,nur dann und nur insoweit als legitim betrachtet [werden], wie sie in widerspruchsfreier Übereinstimmung mit dem Vertragsrecht stehen“ (2009: 343). Doch die Um- und Durchsetzung des Vertragsrechts bedeutet in der Konsequenz eine erhebliche Beschneidung demokratischen Handelns. Insbesondere das europäische Richterrecht richtet sich gegen eine demokratisch legitimierte nationalstaatliche Gesetzgebung, wie Fritz Scharpf (2009: 264 f.) an Beispielen aus dem Sozialund Arbeitsrecht verdeutlicht. Zudem besitzt die Rechtssprechung des Europäischen Gerichtshofes einen deutlichen Bias zugunsten des Abbaus von so genannten Wettbewerbsschranken, also einer vornehmlich „,negativen Integration“ 
(Scharpf 1999: 52 f.) zum Zwecke der Herstellung des Binnenmarktes. Insofern die europäische Rechtssprechung immer mehr Politikfelder unmittelbar oder mittelbar beeinflusst, wird der Gestaltungsspielraum der nationalstaatlichen Gesetzgebung, aber auch des Verwaltungshandelns zunehmend eingeschränkt. Eine positive Integrationspolitik, die hier korrektiv fungieren könnte, ist aufgrund der erheblich höheren Konsensschwellen lediglich marginal ausgebildet. Die daraus resultierende ,Tendenz zur schleichenden Aushöhlung der staatlichen Gesetzgebungsbefugnisse“ (Grimm 2009: 493) hat Konsequenzen für das System der sozialen Sicherungen, das Kräfteverhältnis von Arbeitnehmern und Arbeitgebern sowie die Bereitstellung öffentlicher Güter. In all diesen Bereichen wird die demokratische Selbstgestaltungsfähigkeit durch das europäische Richterrecht und die europäische Komitologie ausgehebelt. Laut Scharpf droht damit nicht nur ein Legitimationsverlust europäischer wie nationalstaatlicher Politik, sondern auf mittlere Sicht auch ein grundlegender Gestaltwandel gesellschaftlicher Strukturen, und zwar in Richtung einer Zweiklassen-Gesellschaft. Ihm zufolge sind ,auf der einen Seite (europaweit operierende) private Versicherungen, private Schulen und Hochschulen und bewachte Ghettos für die Besserverdienenden und auf der anderen Seite die Erosion öffentlicher Sozialleistungen, öffentlicher Dienste und öffentlicher Infrastruktur für diejenigen (...), die für private Lösungen nicht zahlen können“ (Scharpf 2009: 268) nicht unwahrscheinlich.

Um nicht missverstanden zu werden: Es soll der deliberativen Theorie nicht unterstellt werden, dass sie soziale Spaltungsprozesse legitimieren möchte. Verwiesen werden soll aber darauf, dass Rechtfertigungsprozesse nicht in machtfreien Räumen stattfinden und dass Vertragswerke immer auch Machtverhältnisse und hegemoniale Überzeugungen widerspiegeln. Doch die notwendige Verstrickung realer Rechtfertigungsprozesse in politische Macht bleibt ausgeblendet, wenn sie als Ausdruck einer rein prozeduralen Rationalität gedeutet werden. Zumindest in der Konsequenz kommt es dann zu einer Rechtfertigung jener strukturellen Merkmale der europäischen Politik, die zu einem Abbau sozialdemokratischer Errungenschaften führen.

\section{Konzeptionelle Alternativen}

Wir befinden uns mithin in einer demokratietheoretischen Sackgasse. Zwar macht Neyer überzeugend geltend, dass das Beharren auf nationalstaatlichen Legitimationsketten der politischen Realität nicht mehr entspricht. Doch ob eine deliberative Interpretation der postnationalen Konstellation neue Formen 
der Legitimität aufzuzeigen verspricht, die an die Seite demokratischer Legitimitätskonzeptionen treten können, muss zumindest gegenwärtig bezweifelt werden. Denn eine deliberative Umdeutung des Demokratiebegriffs verletzt gerade im Kontext der europäischen Governance sowohl dessen prozedurales Gleichheitspostulat als auch in der Konsequenz das Versprechen der sozialen Inklusion. ${ }^{4}$ Wie könnte eine normativ überzeugende Alternative aussehen? Im Folgenden möchte ich drei theoriepolitische Strategien diskutieren, die jeweils versuchen, auf die postnationale Konstellation eine Antwort zu geben, ohne die zwei Versprechen der Demokratie als normative Leitideen aufzugeben. Eine erste Reaktion beschränkt die Anwendung des Demokratiebegriffs auf politische Systeme, in denen eindeutige und möglichst kurze Legitimationsketten existieren, und plädiert für eine Restaurierung nationalstaatlicher Kompetenzen (a). Die zweite Antwort verabschiedet sich zwar nicht von einem normativ gehaltvollen Demokratiebegriff, bestreitet aber, dass die gegenwärtigen politischen Systeme noch angemessen als Demokratien beschrieben werden können und bevorzugt den Begriff der „Postdemokratie“ (b). Die dritte Antwortstrategie begreift Demokratie nicht länger als eine Herrschaftsordnung, sondern als eine Form des Widerspruchs gegen Verletzungen der Versprechen der Demokratie. Der Einspruch bedarf freilich institutioneller Kanäle, die nicht zu voraussetzungsvoll ausgestaltet werden dürfen (c).

\section{a) Renationalisierung der Politik}

Eine erste Strategie besteht darin, gegen den Trend der Verlagerung politischer Kompetenzen auf die trans- und supranationale Ebene sowie den Trend der Privatisierung politischer Entscheidungen in so genannten „Public-PrivatePolicy-Networks" (Greven 2005) und vielleicht auch gegen den vorauseilenden Gehorsam der politischen und akademischen Eliten auf eine Stärkung der kommunalen, regionalen und vor allem nationalstaatlichen Kompetenzen zu setzen. Diese Strategie besitzt in der gegenwärtigen Diskussion indes nur wenige Befürworter, allzu groß scheint die Angst, als ewig gestriger Nationalist zu gelten. So findet sich auch bei Höreth (2009) bei aller Kritik an der deliberativen Ausdünnung der Demokratie im Kontext der europäischen Gover-

4 An einigen Stellen des Textes erweckt Neyer den Eindruck, es gehe ihm um eine Ersetzung demokratischer Legitimitätskriterien (2009: 335, 349), an anderen Stellen spricht er von der Notwendigkeit einer Neuinterpretation der Demokratie $(336,352)$. Die Strategie, mit Blick auf das System der europäischen Governance nicht mehr von Formen demokratischer Legitimität zu reden, tritt dagegen deutlich in Neyer (2010) hervor. 
nance kein explizites Plädoyer für eine Renationalisierung politischen Entscheidens.

Jüngst hat indes das Bundesverfassungsgericht in seinem Lissabon-Urteil (BVerfG, 2 BvE 2/08) einen Kernbestand an Aufgaben und Strukturen festgelegt, über den nicht in Brüssel und Straßburg, sondern allein in den Mitgliedsländern entschieden werden darf. Dabei handelt es sich um jene Politikfelder, „die die Lebensumstände der Bürger prägen und die in besonderer Weise auf kulturelle, historische und sprachliche Vorverständnisse angewiesen sind“ (249). Als Beispiele führt das BVerfG das Strafrecht, Polizei, Militär, Steuern, Sozialpolitik, Erziehung, Bildung, Medienordnung und den Umgang mit Religionsgemeinschaften auf. In all diesen Bereichen erlaube es das Grundgesetz nicht, Zuständigkeiten so auf die Europäische Union zu übertragen, dass, ,in den Mitgliedstaaten kein ausreichender Raum zur politischen Gestaltung der wirtschaftlichen, kulturellen und sozialen Lebensverhältnisse mehr bleibt" (249). Auch wenn es sich um eine weit auslegbare Formulierung handelt, die Intention der Verfassungsrichter ist deutlich. Es soll sichergestellt werden, dass Kernbereiche politischen Entscheidens in der Verfügungsgewalt einer vorwiegend nationalstaatlich verstandenen demokratischen Souveränität verbleiben. Insgesamt kann sich das BVerfG demokratische Legitimität nur im Nationalstaat vorstellen und geht von einem ,strukturellen Demokratiedefizit“ der Europäischen Union aus, das spätestens dann vorliegt, „,wenn der Kompetenzumfang, die politische Gestaltungsmacht und der Grad an selbständiger Willensbildung der Unionsorgane ein der Bundesebene im föderalen Staat entsprechendes (staatsanaloges) Niveau erreichte“ (264). Dem soll durch die Festlegung eines nationalstaatlichen Kernbestands einerseits und die Stärkung der Mitwirkungsrechte des Deutschen Bundestages andererseits entgegengewirkt werden.

Das Lissabon-Urteil ist nicht zuletzt wegen seiner Betonung der nationalstaatlichen Basis der Volkssouveränität vielfach kritisiert worden. ${ }^{5}$ Und bei der eiligen Verabschiedung der vom Verfassungsurteil eingeforderten gesetzlichen Ausgestaltung der Beteiligungsrechte von Bundestag und Bundesrat hat keine ernsthafte Diskussion darüber stattgefunden, ob und wie sich nationalstaatliche Kompetenzen wieder stärken lassen. Die politischen und akademischen Eliten scheinen größtenteils davon überzeugt zu sein, dass ein derartiges Unterfangen im 21. Jahrhundert nicht, oder nur zu einem sehr geringen Grad, möglich ist. ${ }^{6}$

5 Vgl. als Überblick über die Debatte in der Staatsrechtslehre Ruffert (2009).

6 Davon zeugen die Reaktionen im politischen Feuilleton in den Tagen nach der Verkündigung des Lissabon-Urteils. Vgl. u.a. Schmiese (2009) und Winter (2009). 
Das Bundesverfassungsgericht wirkt mit seinem Lissabon-Urteil mithin wie ein einsamer Rufer in der Wüste. Somit ist nicht davon auszugehen, dass die Strategie einer Renationalisierung spezifischer Kernbereiche politischen Handelns den Trend zur Verlagerung politischer Entscheidungen auf die supranationale Ebene umkehren wird. Doch sollte man bei aller berechtigten Kritik am ,nationalstaatlichen Containerdenken' nicht allzu schnell die postnationale Realität beschwören und diese damit weiter verfestigen.

\section{b) Postdemokratie}

Die Strategie, bei der Suche nach der adäquaten Bezeichnung für moderne westliche Systeme auf den Demokratiebegriff zu verzichten, kann in der Politikwissenschaft vor allem auf die Traditionslinie des ,italienischen Realismus“ von Vilfredo Pareto und Gaetano Mosca über Norberto Bobbio bis zu Danilo Zolo zurückblicken. Folgt man etwa Zolos (1998) Argumentation, dann ist die gegenwärtige Demokratietheorie nicht „realistisch“ genug, wenn sie westliche Gesellschaften allein aufgrund ihrer rechtsstaatlichen Verfasstheit und der Durchführung periodischer Wahlen als ,demokratisch“ bezeichnet. Zolo zufolge ist es angemessener, sie als ,liberale Oligarchien“ zu bezeichnen. Gemeinsam ist diesen Autoren eine grundlegende Skepsis gegenüber einem demokratietheoretischen Diskurs, der seinen Gegenstand mit realen Gesellschaften identifiziert. Eine ähnliche Skepsis wird in einer Reihe von neueren Veröffentlichungen zur „Postdemokratie“" artikuliert. ${ }^{7}$

Laut Colin Crouch (2004) zeichnet sich ein postdemokratisches Regime durch folgende Struktur aus: Die Institutionen der parlamentarischen Demokratie - periodische Wahlen, Wahlkämpfe, Parteienkonkurrenz, Gewaltenteilung - sind auf der einen Seite formal gesehen völlig intakt; und eben dadurch unterscheidet es sich grundlegend von prädemokratischen Gesellschaften. Doch auf der anderen Seite stimme die dahinter stehende Figur der Legitimation politischen Handelns durch die Partizipation des Volkes nicht mehr mit den realen Gegebenheiten überein. Der öffentliche Wahlkampf ist Crouch zufolge ein von rivalisierenden Teams professioneller Spindoctors kontrolliertes Spektakel. Sie bestimmten die politische Agenda durch die Auswahl und anschließende Inszenierung von wenigen Themen, die zudem immer stärker personalisiert werden. Die meisten Bürger spielten lediglich eine passive, stille, bisweilen gar apathische Rolle, unfähig zur eigenen Gestaltung der politischen

7 Als Überblick zur Postdemokratiediskussion vgl. Buchstein/Nullmeier (2006). 
Auseinandersetzung. Im Rücken dieser Inszenierung des ,Wahlspiels' fände der tatsächliche politische Prozess statt, und zwar in Form einer privatisierten Interaktion zwischen gewählten Regierungen und Eliten, die größtenteils die Interessen wirtschaftlich starker Akteure vertreten würden. An die Stelle einer durch Wahlen vermittelten Teilhabe der Bürgerinnen und Bürger an den politischen Entscheidungen sind also laut Crouch intransparente Verhandlungen getreten und der demokratische Prozess diene einzig der Erzeugung von Massenloyalität.

Der Vorteil der Begriffsstrategie von Crouch besteht in ihrem Realismus und dem damit einhergehenden Verzicht, zu hohe Erwartungen an die politischen Systeme des Westens zu richten. Sie ist auch eine polemische Reaktion auf eine gerade unter Politikwissenschaftlern zu beobachtende affirmativ-abgeklärte Haltung ,gegenüber Erscheinungen, die noch vor Kurzem als alarmierende Symptome einer beginnenden Krise der Demokratie gelesen worden wären" (Streeck 2009: 21). Gleichwohl ist die Rede von postdemokratischen Verhältnissen Ausdruck einer Verlegenheit. Altvertrautes verschwindet, doch man weiß nicht so recht, was kommen wird. Denn postdemokratische Systeme zeichnen sich durch ein komplexes und widersprüchliches Nebeneinander von demokratischen und expertokratischen, von staatlichen und privaten, von nationalen und globalen Formen des Regierens aus. Damit ist diesen politischen Regimes eine grundlegende Ambivalenz eingeschrieben: Demokratische Beteiligungsformen werden einerseits eingefordert und in Wahlkämpfen regelmäßig inszeniert, andererseits frustriert, insofern der Gegenstandsbereich des demokratisch zu Entscheidenden schrumpft.

Zudem fällt es offensichtlich schwer, sich von den Versprechen der Demokratie zu lösen. Ein Umstand, der nicht zuletzt die Kritik am Begriff der Postdemokratie motiviert (Richter 2006). Gerade weil demokratische Ideale in den politischen Praktiken von den Parlamentsdebatten bis hin zu Selbstverwaltungsgremien, aber auch in akademischen Diskursen immer wieder beschworen werden, sind wir ihnen so sehr verhaftet. ${ }^{8}$ Um dem Rechnung zu tragen, möchte ich vorschlagen, zwar auf der einen Seite die Diagnose der Postdemokratie als eine deskriptive Kategorie zur Beschreibung der politischen Realität westlicher Gesellschaften zu akzeptieren. Auf der anderen Seite lässt sich der normative Gehalt des Demokratiebegriffes aber dadurch bewahren, dass nicht

8 Dies trifft insbesondere auf eine Politikwissenschaft zu, die sich als „,Demokratiewissenschaft" versteht. Zur Wertgebundenheit der Politikwissenschaft vgl. Voegelin (1959) und Taylor (1975). 
länger politische Systeme, sondern bestimmte politische Handlungsweisen als „demokratisch“ charakterisiert werden, und zwar jene, die sich gegen die Verletzungen der beiden Versprechen der Demokratie richten. Dieser konzeptionelle Vorschlag soll abschließend in Anschluss an Sheldon Wolins „fugitive democracy“ (1996) entfaltet werden.

\section{c) Demokratie als Widerspruch}

Das Konzept der „fugitive democracy“ hat Wolin in Auseinandersetzung mit dem Formwandel der amerikanischen politischen Kultur der 80er und 90er Jahre in einer Reihe von neueren Arbeiten entwickelt. Den Ausgangspunkt bilden eine zeitdiagnostische Beobachtung und eine systematische Annahme. Hinsichtlich der Zeitdiagnose aktualisiert er Tocquevilles Ausführungen zum sanften Despotismus in der Demokratie. In den USA herrsche eine Kultur, in der Freiheit mit dem Recht auf Konsum gleichgesetzt wird, mit der Folge, dass politische Handlungen nur noch als Artikulation individueller Präferenzen wahrgenommen werden. Auf der Strecke bleibe jedoch jenes Moment gemeinschaftlicher Politik, welches Tocqueville als die grundlegende Substanz der amerikanischen Demokratie charakterisiert hat: „What is crucial to the appearance of despotism is the virtual disappearance of the culture of participation and its replacement by a culture of privatism, isolation, and [...] consumerism" (Wolin 2001: 570). Ganz wie Tocqueville es prophezeit hat, handelt es sich laut Wolin um einen milden Despotismus, der die Bürger nicht zwingt, sondern sie auf sanfte Weise in ihrer „,consumer sovereignity“ bestätigt. Es ist der Begriff der „Postdemokratie“, mit dem er diese politische Kultur charakterisiert. Allerdings beschränkt Wolin sich nicht, wie dies tendenziell bei Crouch der Fall ist, auf die Beschreibung postdemokratischer Verhältnisse. Die Diagnose der Postdemokratie ist für ihn vielmehr der Anlass zu einer Neufassung des Demokratiebegriffs.

Ihren Ausgangspunkt besitzt Wolins Neukonzeptionalisierung in der an Max Weber anschließenden Annahme, dass moderne, komplexe Gesellschaften notwendig bürokratische Apparate ausbauen, die kaum Raum für demokratische Selbstregierung lassen: „The political has become specialized, regularized, and administrative in character and quality. Institutionalization marks the attenuation of democracy; leaders begin to appear; hierarchies develop; experts of one kind or another cluster around the centers of decision; order, procedure, and precedent displace a more spontaneous politics" (1996: 39; vgl. 2004). Nun lässt sich gegen diese Diagnose einwenden, dass sie von einem naiven, 
unmittelbaren Demokratieideal ausgeht, dem moderne Flächenstaaten prinzipiell nicht genügen können. Mit Blick auf den Bedeutungszuwachs von Formen der supranationalen oder gar globalen Governance muss man aber feststellen, dass auch die Mechanismen einer repräsentativen Demokratie zunehmend ins Leere laufen. Dies ist ja auch der Anlass für die Vertreter der deliberativen Theorie, nach alternativen Formen der Legitimität zu suchen. Gerade wenn man die Diagnose ernst nimmt, dass eine demokratische Programmierung des Regierungshandelns im nationalstaatlichen Rahmen an Grenzen stößt, gewinnt das Komplexitätsargument mit Blick auf supra-, transund internationale Regelungsarrangements an Überzeugungskraft (Greven 2009: 71). Die neuen Formen der Governance, sofern sie denn in der Lage sein sollen, verbindliche Entscheidungen zu treffen und auch umzusetzen, bedürfen institutioneller Kanäle, die sich aufgrund ihrer Komplexität einer demokratischen Steuerung jedoch größtenteils entziehen. ${ }^{9}$

Wenn sich Demokratie in komplexen Räumen nicht institutionalisieren lässt, dann gewinnt Wolins Vorschlag, Demokratie nicht als eine institutionelle Form oder ein politisches System, sondern als einen Moment des Widerspruches gegen bürokratische Verkrustungen zu konzeptionalisieren, an Attraktivität: „Democracy needs to be reconceived as something other than a form of government: as a mode of being conditioned by bitter experience, doomed to succeed only temporarily, but a recurrent possibility as long as the memory of the political survives“ (1994: 54 f.). Demokratische Momente in diesem Sinne können durchaus von Erfolg gekrönt sein, sie können sich aber laut Wolin nicht auf Dauer stellen, da dies einer Institutionalisierung und damit zwangsläufig einer Machtspaltung gleichkomme. Demokratische Widersprüche seien vielmehr bloß temporäre Eingriffe in den Lauf der Dinge. Derartige demokratische Momente in der Geschichte der Vereinigten Staaten sind ihm zufolge etwa der Kampf gegen die englische Kolonialmacht, die Antisklavereibewegung, die populistischen Bewegungen im 19. Jahrhundert, die Kämpfe zur Einführung des Frauenwahlrechts, die Bürgerrechts- und schließlich die Studentenbewegung gewesen.

Wolin hat sein Model einer "flüchtigen Demokratie“ nicht systematisch entwickelt und schon gar nicht auf die neuen Formen des Regierens bezogen (Kateb 2001; Wiley 2006). Gleichwohl scheint mir die Überlegung, Demokratie nicht als ein politisches System, sondern als eine Handlungsweise zu be-

9 Dies ist der wahre Kern der Diskussion über das Demokratiedefizit der Europäischen Union, welches sich auch durch eine stärkere Parlamentarisierung nicht beheben lässt. 
greifen, geeignet zu sein, die normativen Komponenten des Demokratiebegriffs auch im postnationalen Zeitalter zur Geltung zu bringen. Als ,demokratisch' wären dann insbesondere jene Widerspruchshandlungen zu begreifen, die sich gegen die Verletzungen der beiden Versprechen der Demokratie richten. $\mathrm{Zu}$ bezweifeln ist allerdings, dass es genügt, allein auf die Lebendigkeit politischer Bewegungen zu setzen, wie dies bei Wolin der Fall ist. Bei den oben genannten Beispielen für demokratische Momente handelt es sich nämlich um historische Ausnahmesituationen, deren Zustandekommen sich nicht zuletzt aus einer gemeinsam geteilten politischen Kultur erklärt. Doch auf trans- und supranationaler Ebene kann eine solche Kultur nicht oder zumindest noch nicht vorausgesetzt werden. Um dennoch die Kernidee von Demokratie als Widerspruchshandlungen gegen die Verletzungen der Versprechen der Demokratie in einem postnationalen Kontext plausibel zu machen, möchte ich abschließend eine eher beiläufige Bemerkung von Wolin aufgreifen und weiterentwickeln.

Mit Blick auf die Praxis der antiken Demokratie bezeichnet er das Rotationsprinzip und das Losverfahren als institutionelle Mechanismen, die auf eine Begrenzung der durch Institutionalisierungen hervorgerufenen Machtungleichgewichte zielen: „Rotation and lot [...] are, paradoxically, institutions that subvert institutionalization“ (1994: 43). Diese Verfahren verhinderten die Entstehung einer Oligarchie. Doch das war nicht der einzige Effekt des Rotationsund Losprinzips. So verweist Josiah Ober darauf, dass insbesondere die eher administrative Tätigkeit im athenischen Rat der 500 zusammen mit der lokalen Basis der Demokratie in den einzelnen Demen zu einer Erhöhung und zu einer Verbreitung politischer Kompetenzen in der Bürgerschaft beigetragen hat. „Government ceases to be regarded as a black box, and councilors can quite quickly become fairly expert at the work of politics" (2008: 151). Gewiss, uns trennen Welten von der attischen Demokratie. Doch mit einer gewissen institutionellen Phantasie lassen sich durchaus Mechanismen auffinden, die den Zielen der Verhinderung der Entstehung einer , liberalen Oligarchie“ (Zolo 1998: 219) und der Verbreitung politischer Kompetenzen auch in der heutigen postnationalen Welt entgegenkommen.

Überblickt man die politikwissenschaftliche Literatur der vergangenen Jahre, so stößt man insbesondere auf zwei Vorschläge, die beide Anforderungen zu erfüllen versprechen und zumindest illustrativ erwähnt werden sollen. Da ist zum einen Hubertus Buchsteins (2009) Plädoyer für eine Wiederbelebung des Losverfahrens, gerade auch im Rahmen der Europäischen Union, zu nennen. Konkret schlägt er ein „House of Lots“ als zweite Kammer vor, bestehend aus 
200 Mitgliedern, die alle zweieinhalb Jahre aus den wahlberechtigten Einwohnern der 27 Mitgliedsländer ausgelost werden. Der Reiz dieses Vorschlages besteht darin, dass nicht politische Eliten oder Aktivisten, sondern eine statistisch repräsentative Auswahl der europäischen Bürgerschaft damit zu einer wichtigen Stimme im Gesetzgebungsverfahren erhoben wird. Das „House of Lots" soll nicht nur Empfehlungen aussprechen dürfen, sondern besitzt darüber hinaus auch ein Initiativrecht sowie „ein absolutes Vetorecht“ (Buchstein 2009: 448). Allerdings soll diese zweite Kammer ,ausschließlich auf Legislativakte bezogen sein" (447). $\mathrm{Zu}$ diskutieren ist, ob nicht angesichts des hier vorgeschlagenen Verständnisses von Demokratie als Artikulation von Widerspruch einer derartigen Loskammer nicht auch effektive Kontrollrechte mit Blick auf die Zusammensetzung und Arbeit der europäischen Kommission zugesprochen werden sollten. Das Mehrebenensystem der Europäischen Union würde durch diesen Vorschlag vielleicht an Komplexität zunehmen. Doch könnte dies auch dazu beitragen, deren Legitimationsbasis zu verbreitern und somit die Macht nationaler Veto-Spieler zu schwächen.

Zum anderen hat Heidrun Abromeit (2002: 184 f.), ebenfalls mit Blick auf die Europäische Union, mit dem fakultativen Referendum einen Vorschlag unterbreitet, den sie explizit als eine Form des demokratischen Widerspruchs begreift. Es handelt sich um ein „Veto-Instrument“, das auf eine Entscheidungsblockade in den Fällen zielt, in denen sich die politischen Eliten zu sehr vom demokratischen Souverän entfernt haben. In gewisser Weise können die Voten der niederländischen, französischen und irischen Bürger gegen den Vertrag von Lissabon als derartige negative Referenden aufgefasst werden. Mit ihnen ist ein Widerspruch gegen einen Elitenkonsens artikuliert worden, der zumindest eine aufschiebende Wirkung hatte. Hervorzuheben ist, dass es sich nicht um einen „von oben“ gesteuerten Prozess, sondern um ,einen erstaunlich diskursiven Wahlkampf" (Brunkhorst 2007: 324) gehandelt hat, der von vielen zivilgesellschaftlichen Gruppen getragen worden ist. Sicherlich ist eine kontextsensible Institutionalisierung erforderlich, die verhindert, dass Referenden auf supranationaler Ebene zu einem Instrument von gut organisierten Minderheiten werden. Doch gerade in einem politischen Gebilde wie die Europäische Union, die sich durch eine heterogene Bevölkerungsstruktur und eine hohe Interessenpluralität auszeichnet, könnten Formen der direkten Demokratie im Sinne des Widerspruchs zu einer stärkeren Berücksichtigung der Versprechen der Demokratie beitragen als dies gegenwärtig der Fall ist. 
Die Versprechen der Demokratie und die Grenzen der Deliberation

\section{Literatur}

Abromeit, Heidrun, 2002: Wozu braucht man Demokratie? Die postnationale Herausforderung der Demokratie, Opladen.

Bohman, James, 2007: Democracy across Borders. From Demos to Demoi, Cambridge, MA.

Brunkhorst, Hauke, 2007: Zwischen transnationaler Klassenherrschaft und egalitärer Konstitutionalisierung. Europas zweite Chance, in: Peter Niesen/ Benjamin Herborth (Hrsg.), Anarchie der kommunikativen Freiheit. Jürgen Habermas und die Theorie der internationalen Politik, Frankfurt a. M., 321349.

Buchstein, Hubertus, 2009: Demokratie und Lotterie. Das Losverfahren als politisches Instrument von der Antike bis zur EU, Frankfurt a. M.

Buchstein, Hubertus/Jörke, Dirk, 2003: Das Unbehagen an der Demokratietheorie, in: Leviathan 31 (4), 470-495.

Buchstein, Hubertus/Nullmeier, Frank, 2006: Einleitung: Die PostdemokratieDebatte, in: Forschungsjournal Neue Soziale Bewegungen 19 (4), 16-22.

BVerfG 2009: Urteil vom 30.06.2009, 2 BvE 2/08 u.a. (Vertrag von Lissabon), abgedruckt u.a. in: Neue juristische Wochenschrift 62 (31), 2267-2295.

Christiano, Thomas, 1996: The Rule of the Many. Fundamental Issues in Democratic Theory, Boulder, CO.

Connolly, William E., 1993: The Terms of Political Discourse, Princeton (3. Aufl.).

Crouch, Colin, 2004: Post-Democracy, Cambridge.

Dryzek, John, 2006: Global Deliberative Politics, Cambridge.

Dunn, John, 2005: Setting the People Free. The Story of Democracy, London.

Embacher, Serge, 2009: Demokratie! Nein danke? Demokratieverdruss in Deutschland. Die neue Studie der Friedrich-Ebert-Stiftung, Berlin.

Forst, Rainer, 2007: Das Recht auf Rechtfertigung. Elemente einer konstruktivistischen Theorie der Gerechtigkeit, Frankfurt a. M.

Gallie, Walter B., 1956: Essentially Contested Concepts, in: Proceedings of the Aristotelian Society 56, 167-198.

Goodin, Robert E., 1986: Laundering Preferences, in: Jon Elster/Aanund Hylland (Hrsg.), Foundations of Social Choice Theory, Cambridge, MA., 75101.

Greven, Michael Th., 2005: The Informalization of Transnational Governance. A Threat to Democratic Government, in: Edgar Grande/Louis W. Pauly (Hrsg.), Complex Sovereignity, Toronto, 261-284. 
Greven, Michael Th., 2009: War die Demokratie jemals „Modern“? Oder: des Kaisers neue Kleider, in: Berliner Debatte Initial 20 (3), 67-73.

Grimm, Dieter, 2009: Das Grundgesetz als Riegel vor einer Verstaatlichung der Europäischen Union. Zum Lissabon-Urteil des Bundesverfassungsgerichts, in: Der Staat 48 (4), 475-495.

Habermas, Jürgen, 1971: Vorbereitende Bemerkungen zu einer Theorie der kommunikativen Kompetenz, in: Jürgen Habermas/Niklas Luhmann (Hrsg.): Theorie der Gesellschaft oder Sozialtechnologie. Was leistet die Systemforschung? Frankfurt a. M., 101-141.

Habermas, Jürgen, 1998: Die postnationale Konstellation, Frankfurt a. M.

Höreth, Marcus, 2009: Überangepasst und realitätsentrückt. Zur Paradoxie der Theorie der deliberativen Demokratie in der EU, in: Zeitschrift für Politikwissenschaft 19 (3), 307-330.

Kateb, George, 2001: Wolin as a Critic of Democracy, in: Aryeh Botwinick/ William E. Connolly (Hrsg.), Democracy and Vision, Princeton, 39-57.

Maus, Ingeborg, 2007: Verfassung oder Vertrag. Zur Verrechtlichung globaler Politik, in: Peter Niesen/Benjamin Herborth (Hrsg.), Anarchie der kommunikativen Freiheit. Jürgen Habermas und die Theorie der internationalen Politik, Frankfurt a. M., 350-382.

Münch, Richard, 2009: Transnationale Rationalitäten, nationale Traditionen: ein Dilemma der Regierung im Mehrebenensystem, in: Zeitschrift für Staats- und Europawissenschaften 7 (3-4), 597-621.

Neyer, Jürgen, 2009: Die Stärke deliberativer politischer Theorien und das Elend der orthodoxen Demokratietheorie. Eine Replik auf Marcus Höreth, in: Zeitschrift für Politikwissenschaft 19 (3), 331-358.

Neyer, Jürgen, 2010: Justice, Not Democracy. Legitimacy in the European Union, in: Journal of Common Market Studies 48 (4), 903-921.

Niesen, Peter, 2008: Deliberation ohne Demokratie? Zur Konstruktion von Legitimität jenseits des Nationalstaates, in: Regina Kreide/Andreas Niederberger (Hrsg.), Transnationale Verrechtlichung, Frankfurt a. M., 240-259. Ober, Josiah, 2008: Democracy and Knowledge. Innovation and Learning in Classical Athens, Princeton.

Pettit, Philip, 2004: Depoliticizing Democracy, in: Ratio Juris 17 (1), 52-65.

Richter, Emanuel, 2006: Das Analysemuster der ,Postdemokratie'. Konzeptionelle Probleme und strategische Funktionen, in: Forschungsjournal Neue Soziale Bewegungen 19 (4), 23-37.

Rosanvallon, Pierre, 2009: Democratic Universalism as a Historical Problem, in: Constellations 16 (4), 539-549. 
Die Versprechen der Demokratie und die Grenzen der Deliberation

Rosanvallon, Pierre, 2010: Demokratische Legitimität. Unparteilichkeit - Reflexivität - Nähe, Hamburg.

Ruffert, Matthias, 2009: Nach dem Lissabon-Urteil des Bundesverfassungsgerichts - zur Anatomie einer Debatte, in: Zeitschrift für Staats- und Europawissenschaften 7 (3-4), 381-398.

Schäfer, Armin, 2010: Kompensiert bürgerliches Engagement den Rückgang der Wahlbeteiligung? BBE-Newsletter 4/2010.

Scharpf, Fritz W., 1999: Regieren in Europa: Effektiv und demokratisch?, Frankfurt a. M.

Scharpf, Fritz W., 2009: Legitimität im europäischen Mehrebenensystem, in: Leviathan 37 (2), 244-280.

Schmalz-Bruns, Rainer, 1999: Deliberativer Supranationalismus. Demokratisches Regieren jenseits des Nationalstaates, in: Zeitschrift für internationale Beziehungen 6 (2), 185-244.

Schmiese, Wulf, 2009: „Keine Ohrfeige aus Karlsruhe“, FAZ.net, 01. Juli 2009, http://www.faz.net (Stand: 16.12.2010).

Somek, Alexander, 2009: Demokratie als Verwaltung: Wider die deliberativ halbierte Demokratie, in: Soziale Welt, Sonderheft „Demokratie in der Weltgesellschaft", hrsg. von Hauke Brunkhorst, 323-348.

Streeck, Wolfgang, 2009: Von der gesteuerten Demokratie zum selbststeuernden Kapitalismus. Die Sozialwissenschaften in der Liberalisierung, in: WestEnd. Neue Zeitschrift für Sozialforschung 6 (1), 13-33.

Taylor, Charles, 1975: Erklärung und Interpretation in den Wissenschaften vom Menschen, Frankfurt a. M.

Tocqueville, Alexis de, 1835/40: Über die Demokratie in Amerika, München (1976).

Tucker, Aviezer, 2008: Pre-Emptive-Democracy. Oligarchic Tendencies in Deliberative Democracy, in: Political Studies 56, 127-147.

Urbinati, Nadia, 2010: Unpolitical Democracy, in: Political Theory 38 (1), $65-$ 92.

Voegelin, Eric, 1959: Die Neue Wissenschaft der Politik, hrsg. von Peter J. Opitz, München 2004.

Walter, Franz, 2009: Im Herbst der Volksparteien? Eine kleine Geschichte von Aufstieg und Rückgang politischer Massenintegration, Bielefeld.

Walzer, Michael, 1999: Vernunft, Politik und Leidenschaft. Defizite liberaler Theorie, Frankfurt a. M.

Wiley, James, 2006: Sheldon Wolin on Theory and the Political, in: Polity 38 (2), 211-234. 
Winter, Martin, 2009: Ein Spiel auf Zeit, Sueddeutsche.de, 30.06.2009, http:// www.sueddeutsche.de (Stand: 16.12.2010).

Wolin, Sheldon S., 1994: Norm and Form: The Constitutionalizing of Democracy, in: J. Peter Euben/John R. Wallach/Josiah Ober (Hrsg.), Athenian Political Thought and the Reconstruction of American Democracy. Ithaca, 29-58.

Wolin, Sheldon S., 1996: Fugitive Democracy, in: Seyla Benhabib (Hrsg.), Democracy and Difference, Princeton, 31-45.

Wolin, Sheldon S., 2001: Tocqueville between two Worlds. The Making of a Political and Theoretical Life, Princeton.

Wolin, Sheldon, 2004: Politics and Vision, Expanded Edition, Princeton.

Zolo, Danilo, 1998: Die demokratische Fürstenherrschaft. Für eine realistische Theorie der Politik, Göttingen.

Korrespondenzanschrift:

PD Dr. Dirk Jörke

Institut für Politik- und Kommunikationswissenschaft

Baderstr. 4-7

17489 Greifswald

E-Mail: joerke@uni-greifswald.de 